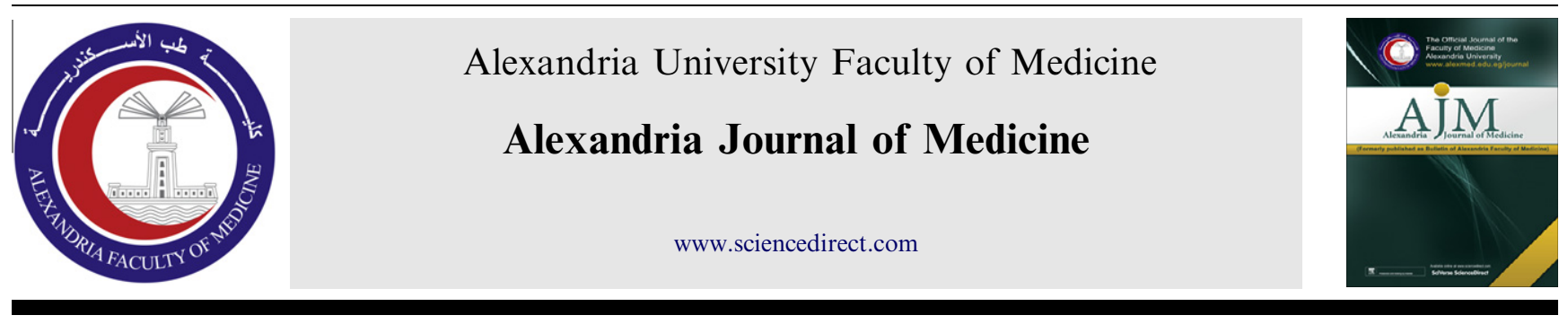

ORIGINAL ARTICLE

\title{
Value of diffusion weighted magnetic resonance imaging in the prediction of cancer prostate
}

\author{
Ahmad Hafez Ahmad Alsayed Afifi ${ }^{\text {a,* }}$, Ashraf Naguib Etaby ${ }^{\text {a }}$ \\ Mohamad Alsayed Yousef Ahmad ${ }^{\text {b }}$, Yasmin Tarek Farghaly ${ }^{\text {a }}$
}

${ }^{a}$ Radiology Department, Faculty of Medicine, Alexandria University, Egypt

${ }^{\mathrm{b}}$ Urology Department, Faculty of Medicine, Alexandria University, Egypt

Received 28 July 2012; accepted 26 August 2012

Available online 12 October 2012

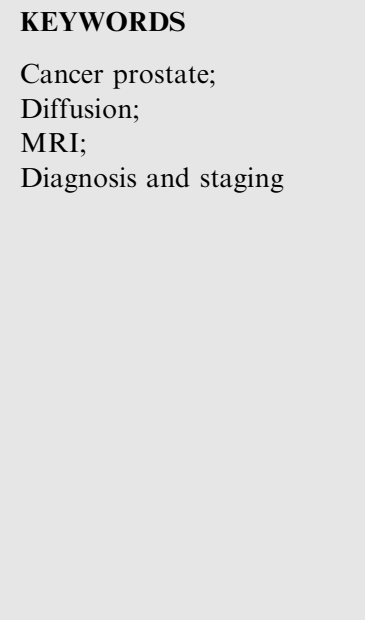

\begin{abstract}
Introduction: Benign prostatic hyperplasia (BPH) and prostatic carcinoma (Pca) are the commonest diseases of the prostate with increased morbidities all over the world along the past few years. Recent studies showed that DWI might be an effective adjunct tool in the localization and potential treatment of prostate cancer with its ability to differentiate the cancer prostate from other benign neoplastic and inflammatory conditions.

Objectives: In the current study we evaluated the role of the diffusion weighted MRI in the detection, localization and staging of cancer prostate.

Methods: Thirty male patients suspected to have prostate cancer were referred from the Urogenital Surgery outpatient clinic to the department of Radio-diagnosis, Alexandria Main University Hospital for radiological evaluation in the time interval from $1 / 1 / 2010$ to $1 / 4 / 2011$. Conventional and DWI MRI studies for evaluation of the prostate were done. For Histopathological evaluation, targeted trans-rectal US-guided biopsies were taken.

Results: According to MRI signals and presentations, in our study, 25 patients had peripheral zone lesions (19 isolated peripheral zone and 6 mixed peripheral and central zones). Regarding central zone lesions ( 5 cases), in four patients proved to have central gland adenocarcinoma and one patient with adenomatous hyperplasia.
\end{abstract}

\footnotetext{
* Corresponding author.

E-mail addresses: a_hafez73@yahoo.com (Ahmad Hafez Ahmad Alsayed Afifi), ettaby777@yahoo.com (A.N. Etaby), youssif_m@ hotmail.com (M.A.Y. Ahmad), a_hafez73@yahoo.com (Y.T. Farghaly).

Peer review under responsibility of Alexandria University Faculty of Medicine.

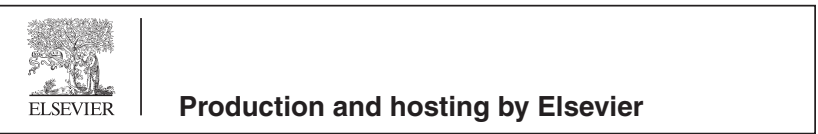


Analysis of data in the receiver operator characteristic curve yielded sensitivity of $100 \%, 95.83 \%$ specificity, 95.83\% PPV, 97\% NPV and $97.87 \%$ accuracy for a cut-off value of $1.2 \times 10^{-3} \mathrm{~mm}^{2} / \mathrm{s}$ for detection of Pca in the PZ. In the CG the sensitivity for detection of Pca foci was $98.2 \%$, specificity $93.24 \%$, PPV $91.67 \%$, NPV $95 \%$ and accuracy $96.8 \%$ for a cut off value of $1.0 \times 10^{-3} \mathrm{~mm}^{2} / \mathrm{s}$. Conclusion: Diffusion MRI is a helpful recent modality in diagnosis and staging of prostatic carcinoma.

(c) 2012 Alexandria University Faculty of Medicine. Production and hosting by Elsevier B.V. All rights reserved.

\section{Introduction}

Prostate cancer is the most common non-cutaneous cancer among males, accounts for $10 \%$ of cancer-related deaths in males and hence, the diagnosis and treatment of prostate cancer continues to evolve. ${ }^{1}$ Of prostate cancer cases, $70 \%$ arise in the peripheral zone, $15-20 \%$ arise in the central zone, and 10 $15 \%$ arise in the transitional zone. ${ }^{2}$ (Table 1 ).

MR imaging is a powerful imaging modality for comprehensive structural evaluation of anatomy and pathology of the prostate gland and seminal tract. Its strengths are related to high soft tissue contrast resolution, high spatial resolution, and a lack of ionizing radiation, whereas the limitations of conventional MRI are related mainly to its cost, insensitivity for the detection of pathology at the molecular level and frequent non specificity of structural imaging abnormalities that are encountered. ${ }^{3}$

Conventional MRI was considered as an excellent technique for the detection of Pca especially by using T2WI which clearly able to distinguish the normal peripheral zone (PZ) and central zone of the gland. ${ }^{4} \mathrm{MR}$ imaging has been found to be more accurate than either DRE or TRUS-guided biopsy for cancer detection and localization. ${ }^{5,6}$ On the other hand, due to the similarity in signal intensity between prostatic carcinoma and benign prostatic hyperplasia on T2-weighted imaging as well as difficult evaluation of transitional zone prostate cancer, the usefulness of conventional MRI is limited. ${ }^{6}$

So benefits of conventional MRI (mainly using T2WI) are predominantly limited to staging for the presence of extracapsular extension and seminal vesicle invasion. ${ }^{5,6}$ In T1-weighted images; the prostate demonstrates homogeneous medium signal intensity and so tumors; are impossible to discern. Hence T1WI is used mainly for the detection of post biopsy hemorrhage to avoid misinterpretation. ${ }^{7}$

Technical advances in MR imaging have led to the development of functional MR imaging techniques such as MR spectroscopy, diffusion Weighted (DWI) MRI, and dynamic contrast enhanced (DCE) perfusion imaging, potentially leading to increased sensitivity, specificity, and accuracy of detection and characterization of disease processes. ${ }^{8}$

By performing DWI (to assess diffusion restriction of the lesion) using different $b$ values, quantitative analysis is possible. There is increasing interest in the application of DWI for detecting post treatment response as, from the MRI diffusion point of view, therapy will result in tumor lysis, loss of cell membrane integrity, increased extracellular space, and, therefore, an increase in water diffusion. ${ }^{8,9}$

\section{Methods}

This antegrade cross sectional study that was done included 30 male patients suspected to have prostate cancer referred from the Uro-genital Surgery outpatient clinic to the department of Radio-diagnosis, Alexandria Main University Hospital for radiological evaluation in the time interval from $1 / 1 / 2010$ to $1 / 4 / 2011$. The provisional clinical diagnosis was based on high PSA level and suspicious digital rectal examination. Patients' ages ranged from 38-83 years with highest incidence in 6th decade. Mean age was $64 \pm 11$ years (SD).

Table 1 Protocols for MRI and DWI.

\begin{tabular}{ll}
\hline MRI scanner & $\begin{array}{l}\text { Siemens (Avanto, Erlangen } \\
\text { Germany) } 1.5 \mathrm{~T} \text { closed magnet } \\
\text { Phased array surface coil TORSO }(16 \\
\text { channels) } \\
\text { Fast spin echo T2 }\end{array}$ \\
- TR $4000 /$ TE 97, Echotrain length 20 \\
- FLIP angle $150^{\circ}$, FOV $220( \pm 20)$ \\
- Matrix size $256 \times 256$, Slice thick- \\
ness 3 mm. No gap used \\
Diffusion & - TR $2100 /$ TE 80, Matrix $128 \times 128$ \\
weighted images: & - Field of view $220( \pm 20)$, slice thick- \\
(EP sequence) & ness 3 mm without gap in between \\
& - $3 b$ values were used $(0,500,1000)$ in \\
& two patients and $(50,400,800)$ in \\
& other 18 patients
\end{tabular}

ADC maps

reconstruction
On workstation (Syngo MR B17) for qualitative and quantitative assessment of DWI images
Philips (Gyroscan Intera-Nederland)1.5

$T$ closed magnet

Phased array surface coil (4 channels)

- TR 3500/TE90

- Flip angle $90^{\circ}$, Field of view $280 \mathrm{~mm}$

- Matrix size 400 , slice thickness $3 \mathrm{~mm}$

- No gap in between

- TR 1000/TE 137, matrix 224

- Field of view $280 \mathrm{~mm}$, flip angle $90^{\circ}$, slice thickness $3 \mathrm{~mm}$ without gap in between

- $3 b$ values $(0,500,1000)$ in three patients and in the other seven patients $b$ values used $(50,400,800)$

On workstation (extended MR work space) for qualitative and quantitative assessment of images 
All examined patients agreed to participate in the study and provided with informed consent to use medical records for research.

Each patient was subjected to full clinical evaluation, DRE, and estimation of prostate specific antigen (PSA) level. Regarding radiological studies; previous imaging modalities including plain KUB, gray scale and power Doppler ultrasonography, IVU, CT or any other imaging modality were checked. Conventional T2 W-MRI followed with DWI, ADC mapping were done.

For Histopathological evaluation, targeted trans-rectal US-guided biopsies were done (from suspicious lesions seen on the DWI either detected on the TRUS and the conventional MRI or not). Also, sextant biopsies (6-12 biopsies) including bilateral upper, middle and lower prostatic zones were done in the Intervention Unit of the Radiology department (12 cases) and in Urology department (18 cases)

\subsection{Technique and parameters}

No specific patient preparation was needed for MRI examination. The patients were laid supine with arms beside the body and the feet were introduced first. Ten patients were examined through a $1.5 \mathrm{~T}$ closed magnet MRI scanner (Gyroscan Intera-Philips medical system-Netherlands) using phased array surface body coil of 4 channels. The other 20 patients were examined through a $1.5 \mathrm{~T}$ closed magnet MRI scanner (Avanto-Siemens-Erlangen-Germany) using phased array surface body coil (TORSO)-16 channels. Scans were obtained from the pelvic inlet to the pelvic outlet. MRI was done before biopsy in all cases. After routine MRI (axial, coronal and sagittal) T2-weighted images, axial diffusion-weighted images were acquired and then ADC values were calculated.

DWI imaging is typically performed with at least two $B$ values $\left(0-1000 \mathrm{~s} / \mathrm{mm}^{2}\right)$ to allow calculation of the ADC. Imaging with low $B$ values provides a higher signal-to-noise ratio (SNR) but with less diffusion weighting. As the $B$ value is increased, sensitivity to the effects of diffusion increased, but at the expense of a longer echo time, a worse SNR, and greater image distortion. The lowest mean ADC value was taken after measuring the ADC value in different areas. The ROI is fitted in a homogenous area averaging about $0.5 \mathrm{~cm}^{2}$ (changeable depending on lesion size). ADC value was measured in the suspected malignant area as well as the normal appearing peripheral zone and normal appearing central zone.

\subsection{Statistical analysis of the data}

Data were analyzed using SPSS software package version 18.0 (SPSS, Chicago, IL, USA). Quantitative data were expressed using Range, mean, standard deviation and median while Qualitative data were expressed in frequency and percent. Qualitative data were analyzed using Mann Whitney test for comparing two groups.

Pearson coefficient was used to analyze the correlation between any two variables. Agreement of different predictive with outcome was used and was expressed in sensitivity, specificity, positive predictive value, negative predictive value, accuracy and likelihood ratio. Receiver operating characteristic curve (ROC) was done where the area under the ROC curve denotes the diagnostic performance of the test. Area more than
$50 \%$ gives the acceptable performance and area about $100 \%$ is the best performance for the test. $p$ Value was assumed to be significant at 0.05 .

\section{Results}

Digital rectal examination (DRE) was performed to all patients. Seven patients had right sided hard nodule, five patients had left sided hard nodule and the other 18 patients had suspicious rectal examination with no definite nodules felt. DRE findings were used as a guide to the site of the lesion on the MR images.

High PSA level was the main including criterion where it ranged from 2.5 to $468 \mathrm{ng} / \mathrm{ml}$. The mean PSA level was 67.5 $( \pm 12.6) \mathrm{ng} / \mathrm{ml}$, where the largest group had a PSA level less than $10 \mathrm{ng} / \mathrm{ml}$ ( 9 patients). Only one patient had a PSA level of $2.5 \mathrm{ng} / \mathrm{ml}$ and he was included in the study because of suspicious DRE and unrelieved LURTS. Two patients had upper limit of the normal value $(5 \mathrm{ng} / \mathrm{ml})$.

After laboratory, clinical and imaging evaluation, prostate cancer was detected in 27 patients $(90 \%)$, adenomatous hyperplasia in two patients $(6.6 \%)$ and prostatitis $(3.3 \%)$ in one patient. The majority of the lesions (19 patients) were in the peripheral zone, in five patients there were isolated central gland lesions while in six patients the lesions were seen involving both zones.

Conventional MRI was done for all patients (mainly screening T2WI) to depict the hypointense area in the peripheral zone or suspicious nodule in the central gland, if present, and correlated it with DW images and hence localized areas needed for measuring ADC values.

According to MRI signals and presentations, in our study, 25 patients had peripheral zone lesions (19 isolated peripheral zone and 6 mixed peripheral and central zones), 22 of them showed hypointense T2 signal compared to the hyperintense peripheral zone and proved to be adenocarcinoma, other one had hypointense signal on T2WI in the peripheral zone and revealed to be prostatitis (false positive case) as well as the remaining two was proven to be adenocarcinoma and they did not appear on the T2WI.

Regarding central zone lesions, in three patients proved to have central gland adenocarcinoma, the lesion was not depicted on T2WI (false negative) and in one patient had a suspicious ill-defined central gland nodule confirmed by the DWI and the pathology to be adenomatous hyperplasia (again false positive case). One remaining case was depicted to have the central gland well defined hypointense nodule and proved to be malignant with other confirmatory modalities.

In the isolated peripheral zone lesions (19 patients) (example at Figure 1), the commonest finding was the presence of hypointense T2WI lesion on a background of normal hyperintense peripheral zone without characterization of the nature of the lesion in 15 patients. On DWI, 14 of them, proved to be carcinomas, showed restricted diffusion (seen as hyperintense on the highest used $b$ value image, hypointense on the ADC map) with a corresponding low measured $\mathrm{ADC}$ value ranging from $0.5-0.97 \times 10^{-3} \mathrm{~mm}^{2} / \mathrm{s}$. The mean ADC value was 0.73 $( \pm 0.14) \times 10^{-3} \mathrm{~mm}^{2} / \mathrm{s}$. The remaining one case of the hypointense lesions seen on the T2WI, was not restricted as hypointense signals on the DWI and relatively hypointense in $\mathrm{ADC}$ map and the measured ADC value was borderline at 

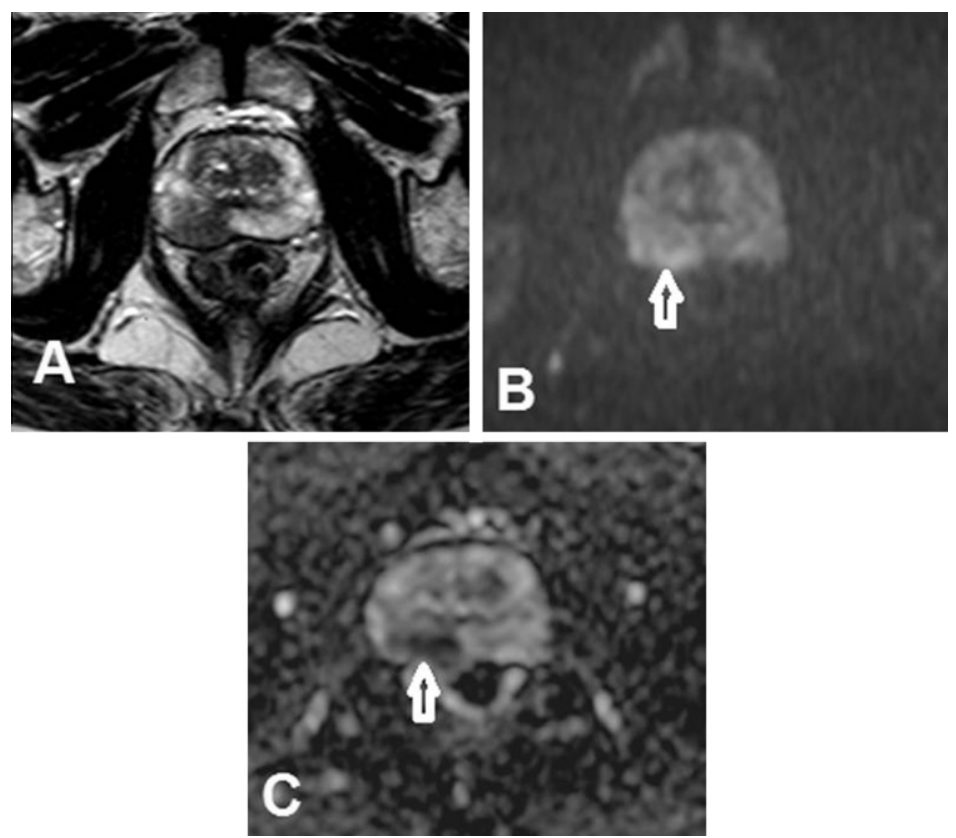

Figure 180 year male had a RT hard nodule felt on DRE. PSA was $9.6 \mathrm{ng} / \mathrm{ml}$. Axial T2WI (A) shows a hypointense right PZ lesion with intact overlying hypointense capsule. The lesion is restricted as hyperintense signals on the axial DWI ( $b$ value 800$)$ (B), hypointense on the ADC map (C) and lowest mean ADC value was $0.68 \times 10^{-3} \mathrm{~mm}^{2} / \mathrm{s}$ (ADC value on the left unaffected PZ was $1.6 \mathrm{~mm}^{2} / \mathrm{s}$ ). Proved after biopsy to be adenocarcinoma Gleason score 6 .

$1.2 \times 10^{-3} \mathrm{~mm}^{2} / \mathrm{s}$. After TRUS guided biopsy it revealed to be granulomatous prostatitis.

DWI depicted two peripheral zone lesions as restricted diffusion with consequently low ADC value which were not seen on the T2WI as they were isointense to the PZ raising the sensitivity of diffusion MRI in depicting lesions compared with routine MR imaging (Figure 2).

Two other patients had hypointense peripheral zone lesions on $T 2 W I$ but their borders, extent and involvement of one or both lobes were ill-defined and they were well defined as restricted area on the DWI with a corresponding low ADC value.

In Central gland lesions ( 5 patients), a hypointense lesion was suspected on $T 2 W I$ on a background of heterogeneous signal of the central gland (characterization on T2WI was difficult as a hyperplastic or a malignant nodule in the presence of heterogeneous gland). However on $D W I$ the lesions were restricted (hyperintense on the highest $b$ value and hypointense
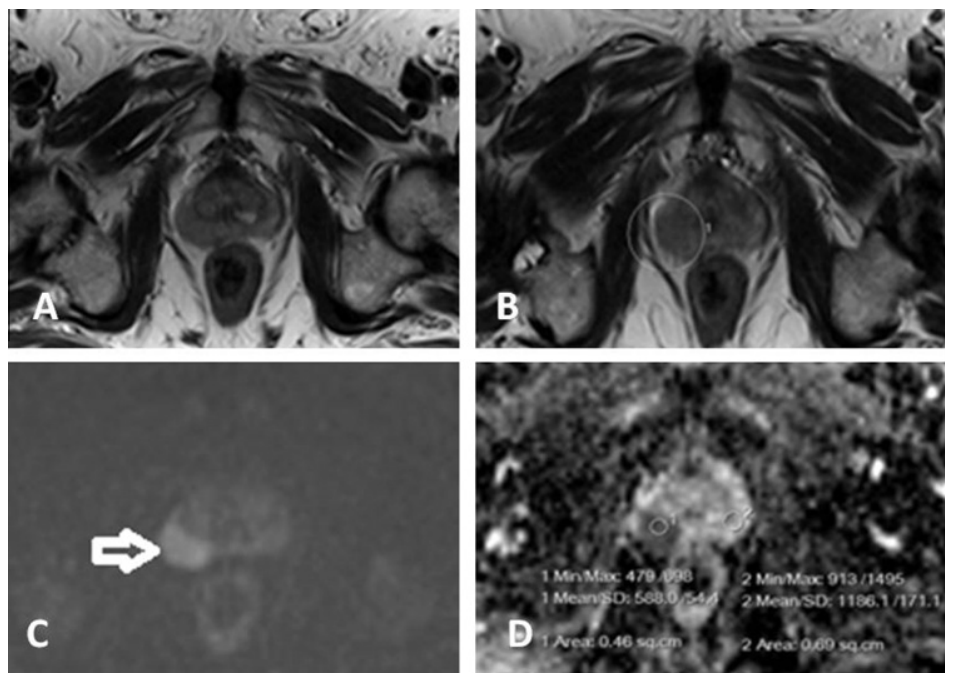

Figure 283 year male had a hard RT nodule by DRE.PSA was $9 \mathrm{ng} / \mathrm{ml}$. Axial T2WI (A and B) showed bulky isointense right PZ (A ROI around the right bulky PZ in (B), which is seen restricted, hyperintense on the axial DWI( $b$ value800) (C), hypointense on the ADC map (D), the lowest mean ADC value was $0.58 \times 10^{-3} \mathrm{~mm}^{2} / \mathrm{s}$ (ROI 1) and the mean ADC value in the left unaffected PZ was $1.2 \times 10^{-3} \mathrm{~mm}^{2} / \mathrm{s}$ Targeted biopsy from RT side was done, proved to be adenocarcinoma Gleason score 8 . 
on the ADC map) and proved histopathologically to be of malignant nature.

DWI depicted another two central gland lesions as restricted diffusion (hyperintense on the DWI and hypointense on the ADC map) with low ADC value which measured 0.76 and $1 \times 10^{-3} \mathrm{~mm}^{2} / \mathrm{s}$. These lesions were seen as intermediate signal on $T 2 W I$, isointense to the surrounding central gland. (Figure
3) DWI excluded malignancy in two other cases proved histopathologically to be adenomatous hyperplasia, the first one showed slightly hyperintensity on the highest $b$ value but also hyperintense on the ADC map ( $T 2$ shine through) with average measured ADC value $\left(1.2 \times 10^{-3} \mathrm{~mm}^{2} / \mathrm{s}\right)$. The other case had a heterogeneous signal of the central gland on $T 2 W I$ but no restricted foci at DW images.
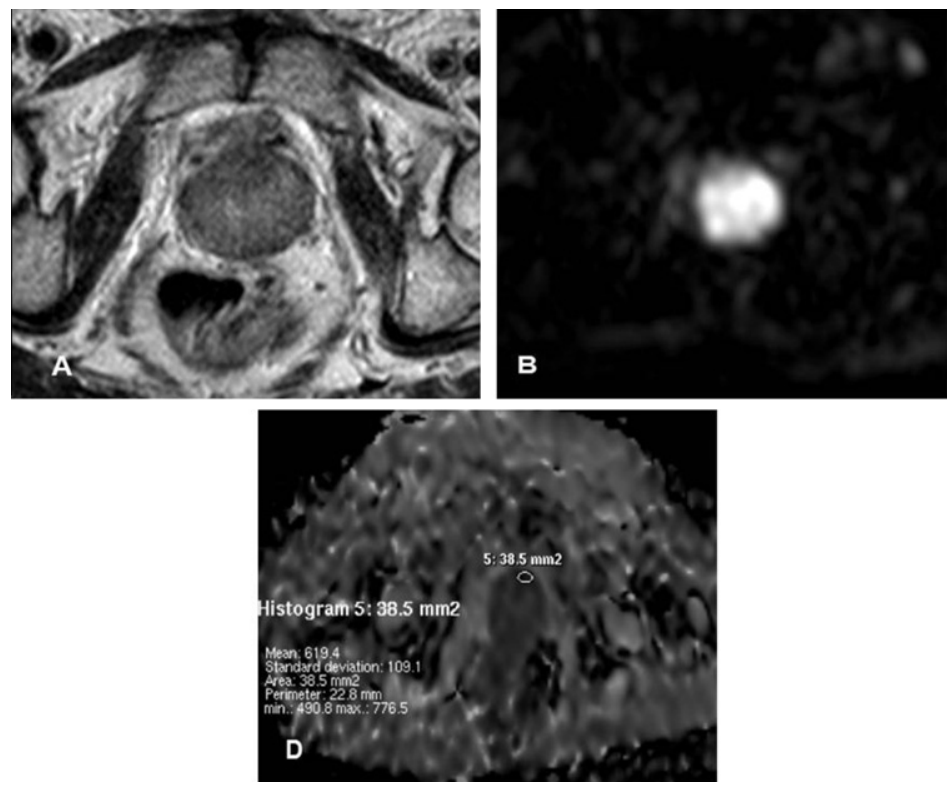

Figure 376 year male with LUT symptoms and PSA was $100 \mathrm{ng} / \mathrm{ml}$. Axial T2WI (A) shows isointense large well defined nodule occupying most of the prostate (mostly originating of the CG), its nature could not be assessed based on the T2WI only. The lesion was restricted, hyperintense on the axial DWI (B) ( $b$ value 1000), hypointense on the ADC map (C). Lowest mean ADC value measured was $0.619 \times 10^{-3} \mathrm{~mm}^{2} / \mathrm{s}$ in the restricted nodule. Proved to be adenocarcinoma Gleason score 9 with perineural invasion.
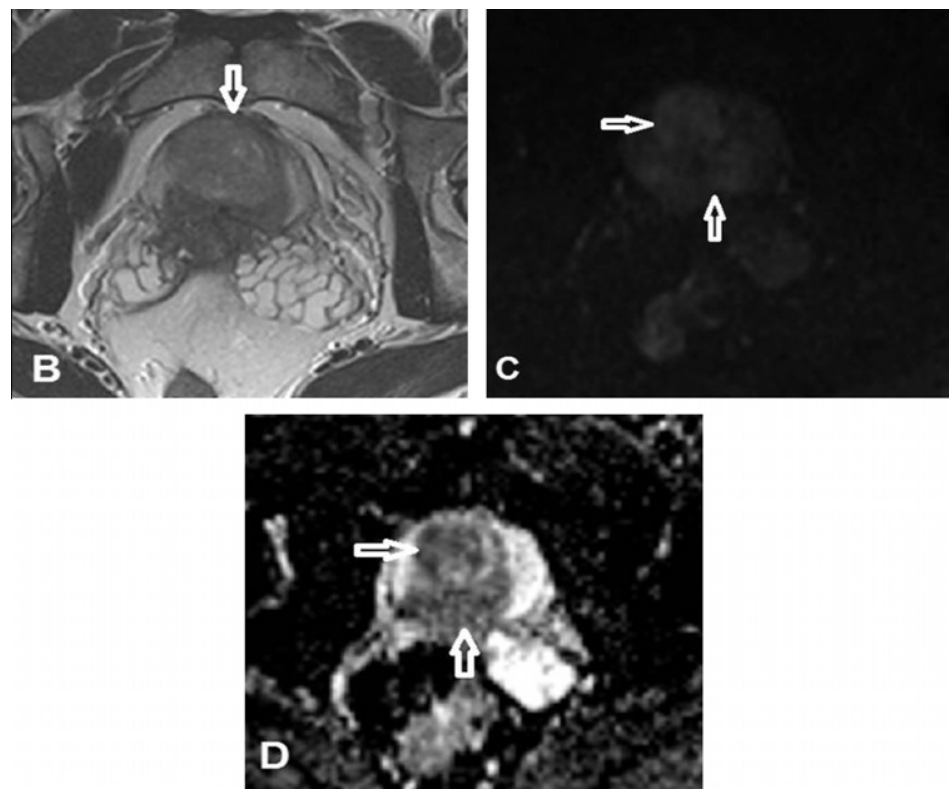

Figure 450 year male patient presented with LURTS, high PSA level $66 \mathrm{ng} / \mathrm{ml}$ and DRE revealed right hard prostatic nodule. Axial T2WI (A) shows hypointensity in the medial parts of both PZs with another well defined iso to hypointense nodule of the CG. Both lesions were restricted, hyperintense on the axial DWI (B) ( $b$ value 800), hypointense on the ADC map (C) with lowest mean ADC value about $0.7 \times 10^{-3} \mathrm{~mm}^{2} / \mathrm{s}$ in both areas. Biopsies obtained by TRUS showing adenocarcinoma Gleason score $6(3+3)$. 
In Combined peripheral and Central gland lesions (6 patients) where the peripheral zone component was depicted as hypointensity on T2WI while central gland involvement was seen as hypointensity continuous with the peripheral zone lesion in two patients (Figures 4 and 5) and isointense in four patients (Figure 6). DWI confirmed restricted diffusion in those cases with low ADC value which were confirmed to be prostatic adenocarcinoma.

DWI depicted Pca foci as restricted diffusion (hyperintense on the highest used $b$ value image and hypointense on the ADC map, which was seen in 25 out of 27 patients with proved Pca (e.g. Figures 1 and 2). However, in two other patients with proved Pca there was no evident hyperintensity on the DWI but still hypointense on the ADC map (Fig. 4).

In cases that were proved not to be malignant, no hyperintensity was seen on the DWI in two patients and one patient showed hyperintensity on both DWI and ADC map (T2 shine through).

Technically, the cases done using phased array abdominal surface coil of 4 channels on the Philips (Gyroscan-Intera $1.5 \mathrm{~T}$ scanner) provided lower signal to noise ratio compared to the cases done using the 16 channel coil on the Siemens (Avanto $1.5 \mathrm{~T}$ scanner).
Analysis of data in the receiver operator characteristic curve yielded sensitivity of $100 \%, 95.83 \%$ specificity, $95.83 \%$ PPV, 97\% NPV and $97.87 \%$ accuracy for a cut-off value of $1.2 \times 10^{-3} \mathrm{~mm}^{2} / \mathrm{s}$ for detection of Pca in the PZ. In the CG the sensitivity for detection of Pca foci was $98.2 \%$, specificity of $93.24 \%$, PPV of $91.67 \%$, NPV of $95 \%$ and accuracy of $96.8 \%$ for a cut off value of $1.0 \times 10^{-3} \mathrm{~mm}^{2} / \mathrm{s}$.

ADC values obtained from foci of Pca using $b$ values $(0$, 500 , and 000) did not differ significantly from those obtained using $b$ values $(50,400,800)$ ( $p$ value was $0.11,>0.05)$.

An inverse relationship was found between the ADC value and the Gleason score in the current study which can predict a high or low grade tumor. There was a significant statistical difference between the mean ADC value measured for Gleason score $\geqslant 7$ (high grade) and score $\leqslant 6$ (low grade) $(p=0.019)$. The mean ADC value of cases with Gleason score $\geqslant 7$ was $0.67( \pm 0.14) \times 10^{-3} \mathrm{~mm}^{2} / \mathrm{s}$ and for those $\leqslant$ score 6 was 0.81 $( \pm 0.14) \times 10^{-3} \mathrm{~mm}^{2} / \mathrm{s}$.

Signs of extraprostatic extensions were surveyed as interruption of the hypointense $\mathrm{T} 2$ prostatic capsule, involvement of the neurovascular bundles and the denovillier's fascia as well as invasion of the rectum, urinary bladder neck and seminal vesicle. Seminal vesicles' invasion was noted as intra-
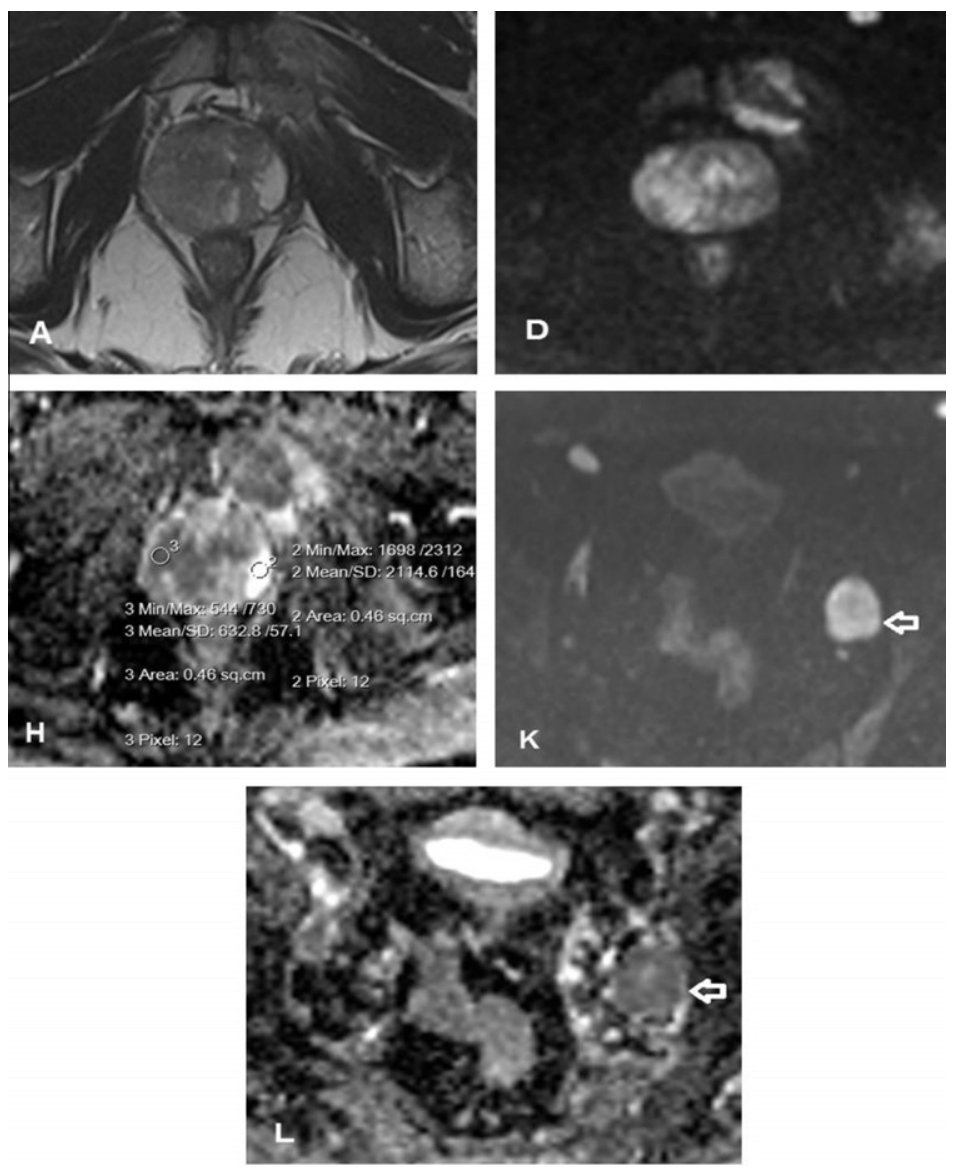

Figure 550 year male patient complained of LURTS and perineal pain. Axial T2WIs (A) shows large hypointense lesion involving most of the PZ, most of the CG and the right SV. The lesions were restricted, hyperintense on the Axial DWIs ( $b$ value 800) (B), hypointense on the ADC map (C). (The diffusion also confirmed involvement of the CG and right SV). The lowest mean ADC value was $0.74 \times 10^{-3} \mathrm{~mm}^{2} / \mathrm{s}$ in $(\mathrm{G})$. Mean ADC value of the spared part of the left PZ was $2 \times 10^{-3} \mathrm{~mm}^{2} / \mathrm{s}$. Axial DWI (D) and ADC map (E) show a restricted left external iliac LN with average ADC value of $0.7 \times 10^{-3} \mathrm{~mm}^{2} / \mathrm{s}$ (confirmed to be malignant node). 
vesicular T2 hypointense signals continuous with the primary tumor. (Figure 7)

Identifying local extensions of a tumor aided in the local staging. If the capsule is not involved which means we are still dealing with a T2 tumor (Figure 1), once the capsule is interrupted this denotes a T3 (T3a unilateral interruption, T3b bilateral interruption (Figure 4) and T3c invading the seminal vesicle (Figure 5). Involvement of the urinary bladder or rectum means a T4 tumor.

Some areas of loco-regional extensions could not be confirmed on the conventional T2WI which was verified on the DWI as restricted diffusion (hyperintensity) on the DWI with a corresponding low ADC value.

\section{Discussion}

Although the sensitivity of T2 weighted images for tumor detection is high, specificity is poor. ${ }^{10}$ Low signal intensity within the peripheral zone on T2-weighted images also can be seen in other non-neoplastic etiologies such as prostatitis, post-biopsy hemorrhage, post-irradiation fibrosis, and following hormonal therapy. ${ }^{10,11}$ Furthermore, the tumors that occur in the central gland $(30 \%)$ are difficult to be detected on T2 weighted imaging because it is not possible to differentiate them from the low signal-intensity and heterogeneous benign nodules of prostatic hyperplasia. ${ }^{12}$

In the current study, we evaluated the role of DWI in the detection of prostate cancer which yielded high sensitivity and specificity, compared to the conventional MRI, in combination with PSA level, DRE and histopathological analysis. High PSA level was the first step for raising suspicion for cancer followed with updated radiological imaging studies, however, 14 patients had PSA level below $20 \mathrm{ng} / \mathrm{ml}, 10$ of them had PSA level below $10 \mathrm{ng} / \mathrm{ml}$. This entails the important role of imaging to detect cancer prostate in the absence of significant PSA elevation. DRE was also positive in 13 patients and was correlated to the images.

Regarding conventional MR images, Gupta et al. ${ }^{13}$ stated that T1WI is used mainly for the detection of post biopsy hemorrhage if biopsy preceded MRI to avoid misinterpretation as prostate cancer, whereas $\mathrm{Yu}$ et al. ${ }^{14}$, stated that $\mathrm{T} 2$ images showed cancer as hypointense area relative to normal peripheral zone tissue due to increased cell density and loss of prostatic ducts.

Recently, Woodfield et al. ${ }^{15}$ stated that, MRI of prostate cancer with conventional T2-weighted imaging is predominantly limited to staging for the presence of extra-capsular extension and seminal vesicle invasion. Concomitantly, Yoshizako et al. ${ }^{16}$ reported that, transition zone cancer detection $(30 \%$ of all prostate cancer) is difficult due to the prevalence of $\mathrm{BPH}$ heterogeneous nodules with undifferentiated $\mathrm{TZ}$ cancer from BPH nodules on T2 or enhanced MRI.

In the current study, conventional T2WI role in the detection of pathology revealed a sensitivity of $88.6 \%$, specificity of $75 \%$, PPV of $95.8 \%$, NPV of $50 \%$ and accuracy for detection was $86.6 \%$. Whereas the study done by Haider et al. ${ }^{17}$ showed that, the sensitivity of T2-weighted imaging alone for the whole prostate was relatively lower at $54 \%$ and specificity was higher at $91 \%$.

Researchers such as Yoshimitsu et al. ${ }^{18}$ reported that, the more recent application of functional MRI techniques, including DWI, has the potential to expand the role of MRI to noninvasive characterization of prostate cancer by providing more specific information regarding tumor location, size, and aggressiveness. He studied 37 cancer prostate patients performed on a $1.5 \mathrm{~T}$ magnet with pelvic phased array surface

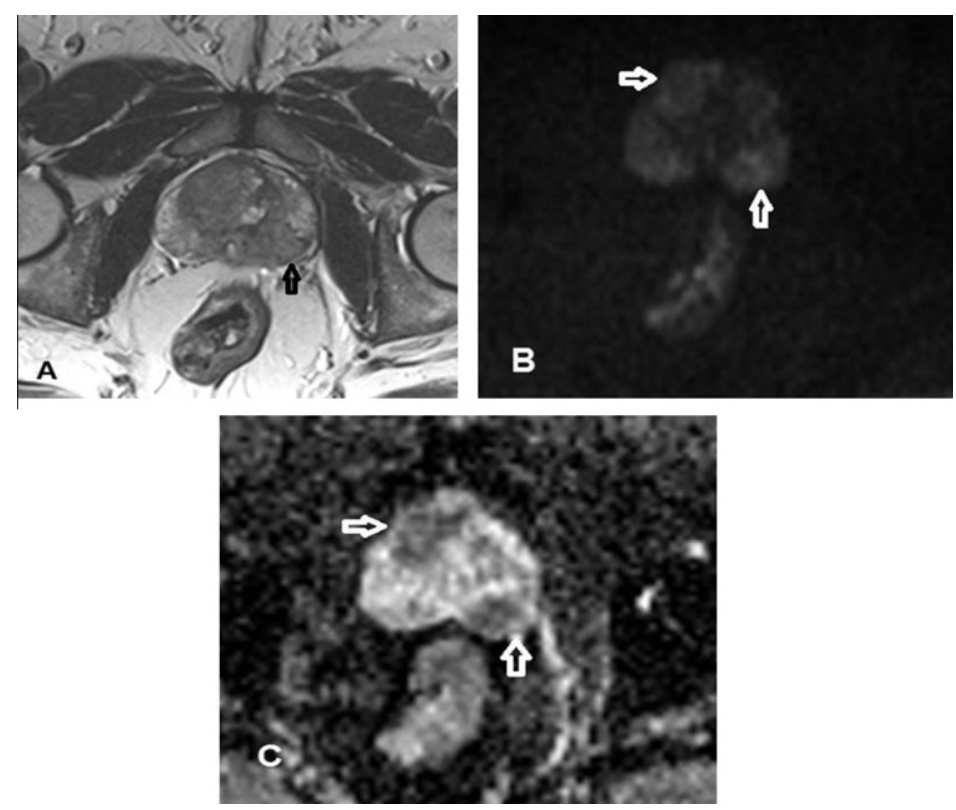

Figure 659 year male patient presented with hard left nodule. PSA was $69.3 \mathrm{ng}$. Axial T2WI (A) revealed left PZ hypointense lesion which corresponds to the findings on DRE, a suspicious bulky isointense nodule to the CG is noted however its character could not be verified on the T2WI only. Both lesions were seen restricted, faintly hyperintense on the axial DWI ( $b$ value 800$)$ (B) hypointense on the ADC map (C) ADC values were respectively $0.57 \times 10^{-3}$ and $0.76 \times 10^{-3} \mathrm{~mm}^{2} / \mathrm{s}$. Histopathologically proved to be adenocarcinoma Gleason score 6 on the right CG nodule and score of 7 on the left PZ nodule. 

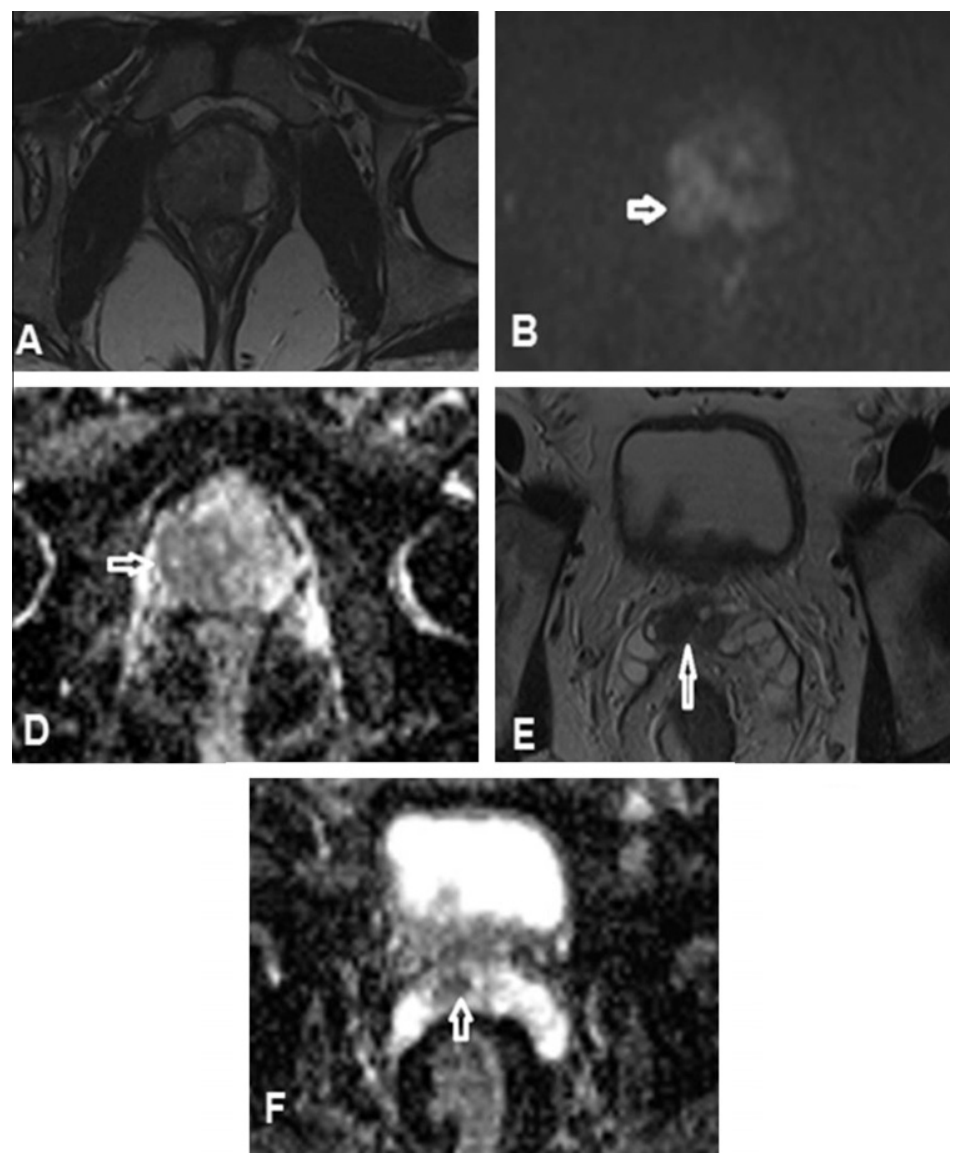

Figure 755 year male patient. Axial T2WI (A), show a hypointense lesion involving the right PZ as well as diffuse hypointensity of the CG. Axial DWIs (B) show restricted diffusion (hyperintensity) of the right PZ with minimal encroachment on the left side of the CG (however the hypointensty involving most of the CG on T2 was not restricted). ADC map (C) reveals hypointensity of the previously noted lesion. The lowest mean ADC value was $0.73 \times 10^{-3} \mathrm{~mm}^{2} / \mathrm{s}$. Axial T2WI (D) shows a hypointense focus at the right SV (suspected invasion) which was confirmed as restricted diffusion on the ADC map (E).

coil, using $b$ values of 0,500 , and $1,000 \mathrm{~s} / \mathrm{mm}^{2}$, despite resulting in images with lower SNR, which can improve lesion detection.

In this study, $B$ values initially used were $0,500,1000$ in five patients aiming at suppression of all the background signals to delineate the restriction however the signal to noise ratio was low and measurements of ADC were difficult in the image due to low signal. Then in the rest of the 25 patients we used the $b$ value of $50,400,800$ aiming at a better signal to noise ratio which also provided good suppression of the background signal with good delineation of the restriction.

On the other hand, Tamada et al. ${ }^{19}$ in their series of patients with prostate cancer, imaging was performed on a 1.5 $\mathrm{T}$ magnet without the use of an endorectal coil and using $800 \mathrm{~s} / \mathrm{mm}^{2}$ as the highest $b$ value.

We performed DWI technique without breath holding, using a multi-channel phased array surface coil only, whereas rectal coil was not used except in one patient who did not tolerate it resulting in motion artifacts. This agreed with Husband et al. ${ }^{20}$ On the other hand, Kim et al. ${ }^{21}$ reported that endorectal coil provides a superior signal-to-noise ratio (SNR) but causes reduced patient compliance and increased susceptibility effects, whereas, the pelvic phased-array coil results in a relatively inferior SNR, which may be overcome by increasing the number of averages used in imaging, however, this compensatory strategy results in a longer image acquisition time. Also, Turkeby et al. ${ }^{22}$ added that endorectal coils provide large gains in signal with reductions in noise, most noticeably at $1.5 \mathrm{~T}$; however, endorectal coils are uncomfortable and expensive.

The considered criterion for the diagnosis of cancer prostate in our study was hyperintense lesion on DWI on

Table 2 Comparison between ADC values in malignant lesions and in normal peripheral and central zones.

\begin{tabular}{|c|c|c|c|}
\hline ADC value & Lesion & Peripheral zone & Central gland \\
\hline Number & 27 & 24 & 21 \\
\hline Range & $0.50-1.1$ & $1.20-2.0$ & $1.0-1.50$ \\
\hline Mean \pm SD & $0.74 \pm 0.15$ & $1.57 \pm 0.19$ & $1.23 \pm 0.14$ \\
\hline Median & 0.74 & 1.55 & 1.20 \\
\hline$Z_{1}(\mathrm{p})$ & & $6.129(<0.001)^{*}$ & $5.839(<0.001)^{*}$ \\
\hline$Z_{2}(\mathrm{p})$ & & & $4.951(<0.001)$ \\
\hline
\end{tabular}


Table 3 Summary of the combination between MRI T2 signals, diffusion restriction and histopathological correlation.

\begin{tabular}{|c|c|c|c|}
\hline Lesions & T2 WI signals & Restriction in DWI & $\begin{array}{l}\text { Histo-pathological } \\
\text { correlation }\end{array}$ \\
\hline $\begin{array}{l}\text { Peripheral zone lesions } \\
\text { (19 patients) }\end{array}$ & $\begin{array}{l}14 \text { Hypointense } \\
2 \text { Ill def. margins hypointense } \\
1 \text { Hypointense }\end{array}$ & $\begin{array}{l}\text { Restricted diffusion } \\
\text { Restricted well defined in diffusion } \\
\text { Not restricted }\end{array}$ & $\begin{array}{l}\text { Adenocarcinoma } \\
\text { Adenocarcinoma } \\
\text { Granulomatous } \\
\text { Prostatitis }\end{array}$ \\
\hline $\begin{array}{l}\text { Combined peripheral } \\
\text { and central } \\
\text { zone lesions ( } 6 \text { patients) }\end{array}$ & $\begin{array}{l}2 \text { Not defined } \\
\text { All with peripheral hypo T2 component } \\
\text { where the central part is T2 hypointense in four } \\
\text { patients and isointense in two patients }\end{array}$ & $\begin{array}{l}\text { Restricted diffusion } \\
\text { All shows restricted diffusion }\end{array}$ & $\begin{array}{l}\text { Adenocarcinoma } \\
\text { All were } \\
\text { Adenocarcinomas }\end{array}$ \\
\hline $\begin{array}{l}\text { Central zone lesions } \\
\text { ( } 5 \text { patients) }\end{array}$ & $\begin{array}{l}1 \text { Hypo intense and two intermediate signals } \\
2 \text { Heterogenous T2 signals }\end{array}$ & $\begin{array}{l}\text { Restricted diffusion } \\
\text { One not restricted, one } \mathrm{T} 2 \text { shine through }\end{array}$ & $\begin{array}{l}\text { Adenocarcinomas } \\
\text { Adenomatous } \\
\text { hyperplasia }\end{array}$ \\
\hline
\end{tabular}

suppressed background with corresponding low ADC value. Ren et al. ${ }^{23}$ and Koh et al. ${ }^{24}$ reported that in Pca, higher signal intensity on DWI and lower ADC compared with BPH nodule and normal tissue is explained with the replacement of normal tissue (composed of water rich acinar structures) by densely packed malignant epithelial cells within small amount of stroma, associated with increased nucleus-to-cytoplasm ratio, resulting in extremely reduced motility of water molecules.

On the other hand, another recent study by Woodfield et al. ${ }^{15}$ revealed that, the criterion for benign prostate tissue was the absence of hyperintensity on the DWI sequence and absence of hypointensity on T2-weighted images in the peripheral zone at a site of biopsy-proven benign tissue. DWI was able to discriminate normal prostate gland, benign prostatic hyperplasia (BPH) nodules, cysts, and carcinoma as lack of hyperintensity with no hypointensity on ADC maps and average ADC value according to the zone, was considered as benign prostatic tissue (Table 2).

In 24 patients in our study that proved to be cancer prostate had hyperintense foci on the DWI and hypointense on the ADC map with corresponding low ADC value compared to the adjacent peripheral or central glands according to its site. This was true except in two cases that proved to be cancer prostate with no hyperintensity in the DWI but with a corresponding low ADC value, may be due to marked hypointense signal on the T2WI (T2 black out effect).

In our study the mean ADC value measured in normal central zone in 21 patients was $1.23 \pm 0.14 \mathrm{~mm} / \mathrm{s}$ (ranging from $1-1.5 \mathrm{~mm} / \mathrm{s}$ ) and the mean ADC value from the normal peripheral zone measured in 24 patients was $1.57 \pm 0.19$. That was matched with Gibbs et al. ${ }^{25}$ who reported that, the normal central gland of the prostate has a lower ADC than the peripheral zone (Table 3).

Comparison between ADC values obtained from cancer foci, normal peripheral and central glands using a b value of $0,500,1000$ did not differ significantly from those using $b$ of $50,400,800$. Using the $b$ value $0,500,1000$ had a lower ADC value in cancer foci and peripheral zone but was not significant, however in both there was a significant difference between cancer foci and normal peripheral and central glands. There is some overlap between ADC values measured in malignant central zone and in the peripheral zone, may be due to the small number of patients with central gland cancer and some patients were examined with different $b$ values.
In our study addition of DWI to the conventional MR images lead to increased sensitivity and specificity compared to the use of conventional MRI (T2WI only) $96.1 \%$ and $100 \%$ respectively. PPV was $100 \%$, NPV was $80 \%$ and accuracy for detection was $96.6 \%$ for a cut off value of $1.1 \mathrm{~mm} / \mathrm{s}$ to differentiate benign from malignant lesions in a ROC curve.

Haider et al. ${ }^{17}$ had reported that T2-weighted imaging combined with DWI is better than T2-weighted imaging alone as addition of DWI to T2WI improved sensitivity from $54 \%$ to $81 \%$. Kumar et al. ${ }^{26}$ reported that, DWI seems to perform better in localization of prostate carcinoma candidate for biopsy, compared with T2WI (Table 4).

DWI helped in the detection of questionable extracapsular invasion of the seminal vesicles and bladder neck. This was confirmed in 10 patients in our study as areas of restricted diffusion. In elderly men, atrophic changes in the seminal vesicles can render the entire seminal vesicle low in signal on T2weighted imaging, thus interfering with the diagnosis. Also DWI had a role in the confirmation of bony metastatic lesion which appeared to be heterogeneous on T1 and T2WIs, by apparently being restricted and of low ADC value, the ones measured in our study was around $5.5 \mathrm{~mm}^{2} / \mathrm{s}$.

Additional benefit of DWI is the ability to determine indices, which may be important in the assessment of disease response to treatment methods. Conventional assessment by measuring lesion size is insensitive to early treatment-related changes.

The presence of lymph nodal metastases in newly diagnosed patient or recurrent prostate cancer is an important prognostic factor leading to greater risk for progression, distant metastases, and death ${ }^{27-29}$ and that up to $80 \%$ of the lymph node metastases can be located in normal sized $(8 \mathrm{~mm})$ lymph

Table 4 Relation between different $b$ values and ADC values.

\begin{tabular}{llll}
\hline & & \multicolumn{3}{l}{$B$ Value } \\
\cline { 3 - 4 } & & $50,400,800$ & $0,500,1000$ \\
\hline Number & & 24 & 3 \\
ADC value & Range & $0.50-1.10$ & $0.59-0.65$ \\
& Mean \pm SD & $0.76 \pm 0.16$ & $0.62 \pm 0.03$ \\
$Z(\mathrm{p})$ & Median & 0.76 & 0.62 \\
\hline
\end{tabular}


node. ${ }^{30}$ They suggest that the ADC value could be a promising tool for differentiating benign from malignant nodes in prostate cancer patients and are significantly more accurate than size criteria. ${ }^{31}$

Finally, regarding study limitations that can be summarized as non use of endo-rectal coil that promised to yield high resolution MR data, application of different $b$ values ( $b$ value of $0,500,1000$ in five patients and 50,400,800 in 25 patients), targeted biopsies were not done in all patients, comparison with other functional MR imaging such as dynamic contrast-enhanced MRI or MR Spectroscopy was not performed (which yield more accurate detection of prostate cancer).

\section{References}

1. Tamada T, Sone T, Jo Y, Yamamoto A, Yamashita T, Egashira $\mathrm{N}$, et al. Prostate cancer: relationships between postbiopsy hemorrhage and tumor detectability at MR diagnosis1. Radiology 2008;248(2):531.

2. Kundra V, Silverman PM, Matin SF, Choi H. Imaging in oncology from the University of Texas MD Anderson Cancer Center: diagnosis, staging, and surveillance of prostate cancer. $\mathrm{Am}$ $J$ Roentgenol 2007;189(4):830.

3. Hricak H, Choyke PL, Eberhardt SC, Leibel SA, Scardino PT. Imaging prostate cancer: a multidisciplinary perspective1. Radiology 2007;243(1):28.

4. Parker C, Fisher C, Desouza N, Payne G. Diffusion-weighted magnetic resonance imaging: a potential non-invasive marker of tumor aggressiveness in localized prostate cancer. Clin Radiol 2008;63(7):774-82.

5. Desouza NM, Reinsberg SA, Scurr ED, Brewster JM, Payne GS. Magnetic resonance imaging in prostate cancer: the value of apparent diffusion coefficients for identifying malignant nodules. Br J Radiol 2007;80(950):90.

6. Akin O, Sala E, Moskowitz CS, Kuroiwa K, Ishill NM, Pucar D, et al. Transition zone prostate cancers: features, detection, localization, and staging at endorectal MR imaging1. Radiology 2006;239(3):784.

7. Lim HK, Kim JK, Kim KA, Cho KS. Prostate cancer: apparent diffusion coefficient map with T2-weighted images for detection a multireader study1. Radiology 2009;250(1):145.

8. Reinsberg SA, Payne GS, Riches SF, Ashley S, Brewster JM, Morgan VA, et al. Combined use of diffusion-weighted MRI and $1 \mathrm{H}$ MR spectroscopy to increase accuracy in prostate cancer detection. Am J Roentgenol 2007;188(1):91.

9. Miao H, Fukatsu H, Ishigaki T. Prostate cancer detection with 3-T MRI: comparison of diffusion-weighted and T2-weighted imaging. Eur J Radiol 2007;61(2):297-302.

10. Morgan VA, Kyriazi S, Ashley SE. Evaluation of the potential of diffusion-weighted imaging in prostate cancer detection. Acta Radiol 2007;48(6):695-703.

11. Choi YJ, Kim JK, Kim N, Kim KW, Choi EK, Cho KS. Functional MR imaging of prostate cancer1. Radiographics 2007;27(1):63.

12. Shimofusa R, Fujimoto H, Akamata H, Motoori K, Yamamoto $\mathrm{S}$, Ueda T, et al. Diffusion-weighted imaging of prostate cancer. $J$ Comput Assist Tomogr 2005;29(2):149.

13. Gupta E, Torigian DA. MR imaging of the prostate gland. PET Clinics 2009;4(2):139-54.

14. Yu KK, Hricak H. Imaging prostate cancer. Radiol Clin North Am 2000;38(1):59-85.
15. Woodfield CA, Tung GA, Grand DJ, Pezzullo JA, Machan JT, Renzulli JF. Diffusion-weighted MRI of peripheral zone prostate cancer: comparison of tumor apparent diffusion coefficient with Gleason score and percentage of tumor on core biopsy. Am J Roentgenol 2009;194(4):W316.

16. Yoshizako T, Wada A, Hayashi T, Uchida K, Sumura M, Uchida $\mathrm{N}$, et al. Usefulness of diffusion-weighted imaging and dynamic contrast-enhanced magnetic resonance imaging in the diagnosis of prostate transition-zone cancer. Acta Radiol 2008;49(10):1207-13.

17. Haider MA, van der Kwast TH, Tanguay J, Evans AJ, Hashmi $\mathrm{G}$, Lockwood $\mathrm{G}$, et al. Combined T2-weighted and diffusionweighted MRI for localization of prostate cancer. Am J Roentgenol 2007;189(2):323.

18. Yoshimitsu K, Kiyoshima K, Irie H, Tajima T, Asayama Y, Hirakawa M, et al. Usefulness of apparent diffusion coefficient map in diagnosing prostate carcinoma: correlation with stepwise histopathology. J Magn Reson Imaging 2008;27(1):132-9.

19. Tamada T, Sone T, Jo Y, Toshimitsu S, Yamashita T, Yamamoto A, et al. Apparent diffusion coefficient values in peripheral and transition zones of the prostate: comparison between normal and malignant prostatic tissues and correlation with histologic grade. $J$ Magn Reson Imaging 2008;28(3):720-6.

20. Husband JE, Padhani AR, MacVicar AD, Revell P. Magnetic resonance imaging of prostate cancer: comparison of image quality using endorectal and pelvic phased array coils. Clin Radiol 1998;53(9):673-81.

21. Kim CK, Park BK. Update of prostate magnetic resonance imaging at 3 T. J Comput Assisted Tomogr 2008;32(2):163.

22. Turkbey B, Albert PS, Kurdziel K, Choyke PL. Imaging localized prostate cancer: current approaches and new developments. $\mathrm{Am} J$ Roentgenol 2009;192(6): 1471.

23. Ren J, Huan Y, Wang H, Zhao HT, Ge YL, Chang YJ, et al. Diffusion-weighted imaging in normal prostate and differential diagnosis of prostate diseases. Abdom Imaging 2008;33(6):724-8.

24. Koh DM, Collins DJ. Diffusion-weighted MRI in the body: applications and challenges in oncology. Am $J$ Roentgenol 2007; 188(6): 1622 .

25. Gibbs P, Liney GP, Pickles MD, Zelhof B, Rodrigues G, Turnbull LW. Correlation of ADC and T2 measurements with cell density in prostate cancer at 3.0 Tesla. Invest Radiol 2009;44(9):572.

26. Kumar V, Jagannathan NR, Kumar R, Thulkar S, Gupta SD, Dwivedi SN, et al. Apparent diffusion coefficient of the prostate in men prior to biopsy: determination of a cut off value to predict malignancy of the peripheral zone. NMR Biomed. 2007;20(5):505-11.

27. Cagiannos I, Karakiewicz P, Eastham JA, Ohori M, Rabbani F, Gerigk C, et al. A preoperative nomogram identifying decreased risk of positive pelvic lymph nodes in patients with prostate cancer. J Urol 2003;170(5):1798-803.

28. Hricak H, Schder H, Pucar D, Lis E, Eberhardt SC, Onyebuchi $\mathrm{CN}$, et al., editors. Advances in imaging in the postoperative patient with a rising prostate-specific antigen level. $J$ Urol 2003;171(3):13-138.

29. Partin AW, Pound CR, Clemens JQ, Epstein JI, Walsh PC. After anatomic radical prostatectomy. The Johns Hopkins experience after 10 years. Urol clin North Am 1993;20(4):713.

30. Heesakkers RAM, Hvels AM, Jager GJ, van den Bosch H, Witjes HPJ, Raat HPJ, et al. MRI with a lymph-node-specific contrast agent as an alternative to CT scan and lymph-node dissection in patients with prostate cancer: a prospective multicohort study. Lancet Oncol 2008;9(9):850-6.

31. Reinsberg SA, Payne GS, Riches SF, Ashley S, Brewster JM, Morgan VA, et al. Combined use of diffusion-weighted MRI and $1 \mathrm{H}$ MR spectroscopy to increase accuracy in prostate cancer detection. Am J Roentgenol 2007;188(1):91-9. 